\title{
Throughput Optimization of Backscatter-Assisted Wireless Relay Networks in Obstacle Environment
}

\author{
Jinfeng Li, Qinyin Ni, and Xiaorong Zhu \\ School of Communications and Information Engineering, Nanjing University of Posts and Telecommunications, \\ Nanjing 210003, China \\ Correspondence should be addressed to Xiaorong Zhu; xrzhu@njupt.edu.cn
}

Received 18 September 2021; Revised 8 December 2021; Accepted 22 December 2021; Published 3 February 2022

Academic Editor: Chunguo Li

Copyright (c) 2022 Jinfeng $\mathrm{Li}$ et al. This is an open access article distributed under the Creative Commons Attribution License, which permits unrestricted use, distribution, and reproduction in any medium, provided the original work is properly cited.

\begin{abstract}
Internet of Things nodes need new charging methods, and RF energy is not well utilized. Backscatter can harvest RF energy from the environment and use the idle spectrum to realize wireless power supply communication. In this article, each enhanced backscatter device (eBD) can select from a variety of cooperative receivers (CR) to provide different transmission services in three modes: energy harvesting (EH), relay communication (RelayCom), and backscatter communication (BackCom). To analyze the throughput of the secondary network, we model the three working modes, respectively. We describe the problem as the objective function of three variables: the period $\alpha$ of $\mathrm{EH}$ when there are no obstacles, period $\mu$ of RelayCom with obstacles, and period $\gamma$ of BackCom with obstacles. The ideal time allocation strategy is then investigated. Then, we apply the particle swarm optimization algorithm to obtain a solution that maximizes the throughput. The simulation results show that compared to the communication network using backscatter or relay protocol alone, the proposed backscatter-assisted wireless relay communication model can greatly improve the throughput and coverage of the secondary system.
\end{abstract}

\section{Introduction}

With the development of the Internet of Things, more and more Internet of Things nodes need to be connected to the network. In smart houses, offices, hospitals, and other scenes, it is not ideal for so many devices to plug in or charge and change batteries constantly. Especially in harsh environments (such as battlefield, forest, ocean, mountains, etc.) or in the field of health care (such as living intelligent medical devices, intelligent contact lenses, wearable gadgets, and implantable neural recording devices), it is unrealistic to replace batteries frequently [1]. On the other hand, with the exponential growth of the number of Internet of Things devices, spectrum congestion is becoming more and more serious, which limits the number of deployable devices and the spectrum efficiency of channels [2]. Network capacity is an essential indicator of the development of the Internet of Things. How to effectively employ the energy of RF resources while also making the service life of energy limited equipment last has become a research focus. Because environmental backscatter does not require additional energy supply, it is a green network communication mode. Environmental backscatter harvests RF energy from the environment and uses free spectrum to realize wireless energy supply communication. It has great potential in addressing energy shortages and spectrum congestion. It is an enabling technology for sustainable communication in the Internet of Things [1]. It is inconvenient to replace the battery or charge the devices, and the backscatter device (BD) can be powered remote; so, wireless energy supply has a strong appeal in the communication of wireless device. In traditional radio networks, backscatter-assisted relay communication can improve the reliability of wireless data transmission in improving the transmission range of $\mathrm{RF}$ signal or avoiding the signal from being blocked by obstacles. The use of a wireless energy supply relay can significantly improve the system performance in terms of energy efficiency and space efficiency [3].

Environmental backscattering converts the RF signal in the environment into electrical energy and transmits data by passively reflecting the existing RF signal in the air. This $\mathrm{RF}$ signal serves as both an energy source and a data carrier 
for a vast number of devices in the Internet of Things [4]. When the main channel is mostly occupied, the BD mainly transmits data by backscatter or harvest energy [5]. When the main channel is always occupied, and BD actively sends information, the transmission power threshold must be adjusted to avoid irreparable interference to the main signal [6]. When the main channel is idle, BD uses the harvested energy to transmit data. The combination of backscatter communication and traditional RF-driven cognitive networks offers enormous promise for enhancing network performance, coping with energy shortage and spectrum scarcity.

Backscatter-assisted wireless communication consumes relatively little power due to the combination of a backscatter transmitter and an energy collection device. It realizes data forwarding and transmission by switching three transmission modes: energy harvesting, active relay transmission, and environmental backscatter. Since the relay is in the active transmission mode and requires higher transmission energy, RF is required to provide sufficient energy. When the energy is scarce, the passive communication method will be used to transmit data and build a hybrid system to make the $\mathrm{BD}$ more powerful and last longer. When there are obstacles between the base station (BS) and the receiver, that is, the direct communication link is blocked, the BS signals can be relayed to the receiver through the BD's relay function. At present, the selection between backscatter communication and relay communication mode is mainly through the power threshold and signal-to-noise ratio, which depends on environmental factors and CSI [6].

At present, there are few studies on environmental backscatter-assisted wireless communication relay in obstacle environments in the literature. According to reference [7], secondary users of wireless energy supply have the ability of environmental backscatter. In this network, when the primary user is transmitting data, there is no open spectrum; thus, the secondary user can perform energy harvesting or environmental backscatter. When the primary user stops transmission, the secondary user can use the harvested energy for active transmission to expand the transmission distance. Reference [6] established the environmental backscatter-assisted communication model and proposed a method to improve the network performance. In this reference, the environmental backscatter communication was combined with the cognitive radio network, and the model was the scene of a single secondary user. Reference [8] constructed a model of hybrid backscatter assisted cognitive wireless network and studied the optimal time allocation between backscatter mode and energy harvesting mode when the channel is busy. It obtained the optimal combination of working modes. Literature [9] analyzed the performance of environmental backscatter communication in RF cognitive radio networks based on random geometry and extended the model in a large range. It studied the coverage probability of the network. Literature [10] studied the dynamic access point (AP) and service selection in backscatter-assisted RF-powered cognitive networks. Without taking into account the randomness of communication channels, the joint AP and service selection problem is stated as an evolutionary game. Literature [11] realized incentive sharing in real-time D2D streaming media networks from the perspective of the mean-field game and studied the cooperative incentive problem in large-scale multiagent systems. By analyzing the current situation at home and abroad, we conclude that if the environmental energy is insufficient or the interference level is high, environmental backscatter is an excellent auxiliary scheme for wireless transmission.

The hybrid communication mode, which combines wireless energy supply active transmission and passive environment backscatter technology, can solve the problem of active transmission's high power consumption [12]. Although environmental backscatter has many advantages, it has a low bit rate because the environmental backscatter transmitter transmits information at a lower Nyquist information rate than the environmental carrier signal and adopts a small series of simple modulation schemes [4]. In addition, environmental backscatter is unsuitable for remote communication due to the round-trip path loss of the environmental carrier signal [13]. The third disadvantage is that the power control of the environmental backscatter transmitter is challenging, and it is not easy to establish a reliable communication connection. This possibility of active and passive communication integration has been confirmed recently by a prototype Briadio [14] with Bluetooth and backscatter functions at the same time.

However, deploying wireless energy supply relay faces some new challenges. Since the relay device with a wireless energy supply involves active transmission, that is, it needs to generate active RF signals, which will require the relay device to use more power-consuming analog circuit elements (such as mixer, amplifier, filter, and oscillator) [4]. As a result, the relay device may take a long time to harvest and accumulate energy to relay the signal, and the time required to maintain the signal relay is relatively short [15]. When the ambient RF signal is weak or intermittent, this situation will become worse. To meet these challenges, certain existing network protocols can make use of network resources more effectively, and some current literature mainly designed these network protocols. For example, energy harvesting then cooperative protocol can be introduced for the relay to maximize the throughput by optimizing the time allocation of downlink energy harvesting and uplink information forwarding [16]. Due to the characteristics of active transmission, the high power consumption of the circuit still limits its performance improvement.

To improve the reliability of backscatter communication and the signal transmission duration of wireless energy supply relay, this paper studies the hybrid mode of backscatter assisted relay communication in obstacle environments. The secondary transmitter in this model is the combination of the backscatter device and the passive relay. It is referred to as an enhanced backscatter device, or eBD for short. The receiver is the combination of the primary and secondary receivers. It is referred to as cooperative receiver, or CR for short. The eBD is equipped with an energy harvesting circuit, a relay circuit, and a backscatter circuit. The energy harvesting circuit harvests RF energy, the relay circuit forwards the signal from the primary transmitter (PT), and the backscatter circuit backscatter the RF signal. The eBD 
in this paper has two characteristics. First, eBD is always within the coverage of PT and is equipped with highcapacity energy storage equipment. Second, only when there are obstacles between PT and CR, eBD relay and forward information is required, which greatly reduces the energy consumption and improves the energy efficiency. We design the mode selection in the hybrid relay and backscatter system into three different operating modes: EH mode, RelayCom mode, and BackCom mode. The main contributions of this paper can be summarized as follows.

(1) This paper designs eBD with three working modes: $\mathrm{EH}$, RelayCom, and BackCom. To achieve the balance of the three modes, the optimal time scheme is studied, and the optimal values of the three variables $\alpha, \mu$, and $\gamma$ are found. The existence, uniqueness, and stability of this equilibrium are proved analytically

(2) We derive the maximum expression of the objective function of the eBD throughput. It is proved that this expression has extreme points and global optimal solution, and the maximum throughputs of the objective function in different environments are obtained

(3) We describe the system throughput maximization problem as a particle swarm optimization problem and propose a three-dimensional parameter solution algorithm based on the particle swarm optimization algorithm. The algorithm has high computational efficiency and strong scalability. It is suitable for multidimensional target search space. The experimental results are in good agreement with the analytical results, which verifies the superiority of the proposed model

The rest of the paper is organized as follows. Section 2 introduces the system model of backscatter-assisted wireless relay communication. Section 3 discusses the analysis models of three working modes. Section 4 outlines the solutions of model optimization of three working modes. Section 5 evaluates the effectiveness of the proposed solution through experiments. Finally, the conclusion and prospect are given in Section 6.

\section{System Model}

This paper assumes the system model depicted in Figure 1 is composed of a master network and slave network. The master network is made up of PT and primary receiver, whereas the slave network is made up of eBD and secondary receiver. Each $\mathrm{eBD}$ corresponds to multiple CRs, each of which includes a primary receiver and a secondary receiver. PT is the energy supply and original signal transmission device of the whole system. The secondary transmitter, eBD, is equipped with a high-capacity energy storage device and performs the roles of EH, RelayCom, and BackCom. CR is capable of receiving the original signal, the backscattered signal, and the relay signal. This paper assumes that there are

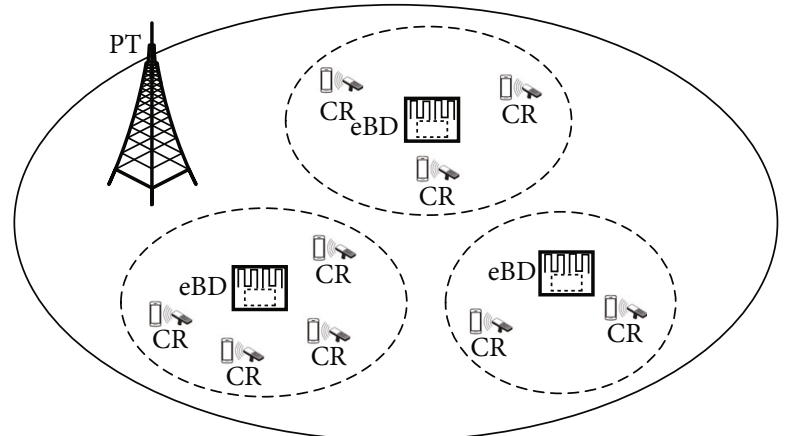

eBD CR

Figure 1: Backscatter-assisted wireless relay communication scenario.

no obstacles between PT and eBD, and there may be obstacles between PT and CR, such as pedestrians, trees, and buildings. When obstacles block the direct communication link, the eBD relays and backscatters the information from PT to CR. When there are no obstacles, PT and CR can communicate directly without eBD forwarding information. The only thing eBD needs to do is backscatter data and harvest energy. Each CR can provide relay and backscatter services for eBD data transmission, and it can choose whether to receive relay signals or backscatter signals.

This paper focuses on the selection and time allocation of EH, RelayCom, and BackCom modes of eBD. When no obstacles exist, eBD needs to balance the time of $\mathrm{EH}$ and BackCom. When there are obstacles, eBD needs to balance the time of EH, RelayCom, and BackCom to ensure the maximum throughput of the secondary system. The key to improving relay performance when using environmental backscatter-assisted relay communication is to pick an appropriate communication mode based on the network environment.

Backscatter-assisted relay communication technology in an obstacle environment provides the functions of expanding data transmission coverage, avoiding obstructions, solving energy shortage, and boosting spectral efficiency. It varies according to different application scenarios and design purposes. In Figure 2, the color arrow indicates three possible working modes of eBD, while the black arrow indicates the communication line of $\mathrm{eBD}$ in another time unit.

No matter whether there are obstacles or not, EH (green arrow in Figure 2) can be carried out to prepare for the RelayCom mode as long as the eBD has no relay or backscatter information. The relay communication mode (red arrow in Figure 2) based on the EH stage can relay forwarding data actively. BackCom mode (blue arrow in Figure 2) can transmit data passively despite obstacles, with the characteristics of slow speed and low energy consumption. eBD is a single antenna structure, and the secondary system transmits data via TDMA. Therefore, a relay device only sends signals to one terminal at the same time. This paper assumes that CR can automatically identify the communication mode of $\mathrm{eBD}$ and use the corresponding demodulator. 


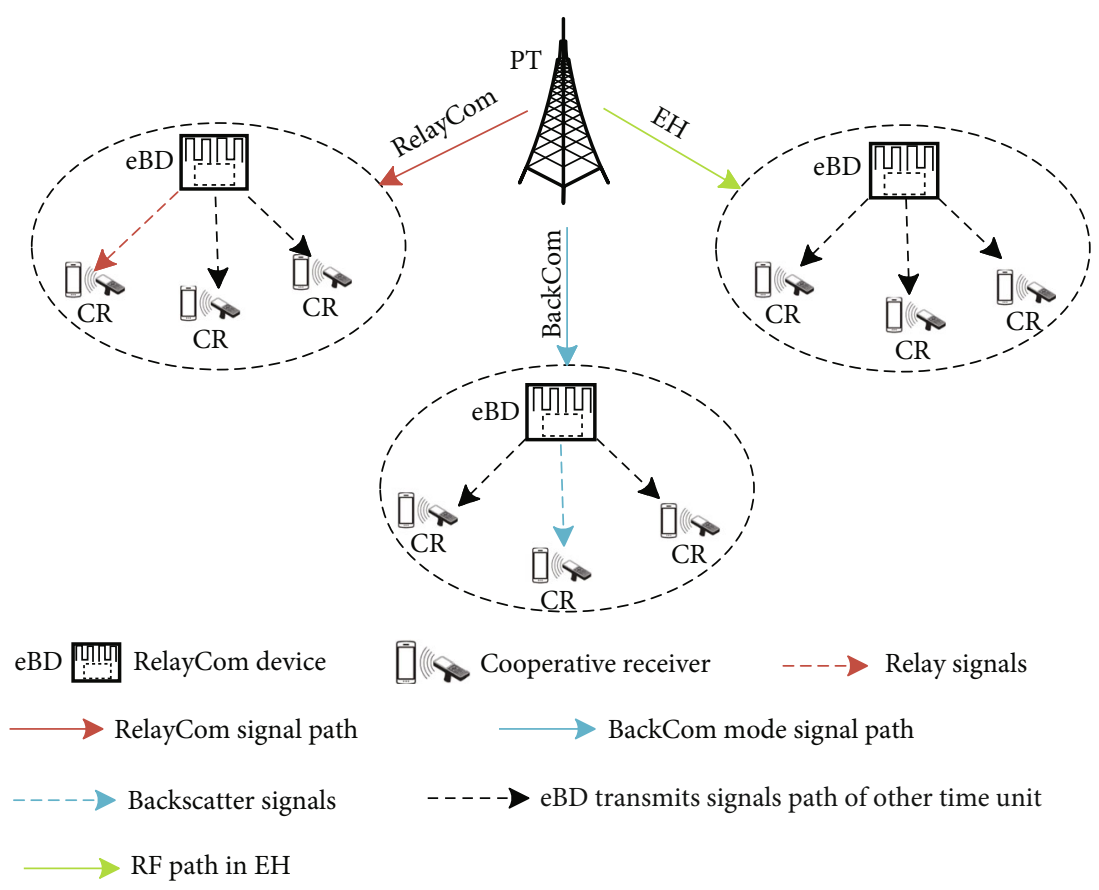

FIgURE 2: Three modes of eBD data transmission.

\section{Analysis Model of Three Working Modes}

Each $\mathrm{CR}$ can provide relay transmission and backscatter transmission services. Of course, it also has the potential to spread to more cases. Following that, the three working mode models will be analyzed in detail, and the throughput expressions of eBD under different conditions are derived.

As illustrated in Figure 3, the ambient backscatterassisted wireless relay communication is divided into two cases: with obstacles and without obstacles, the period with obstacles is $\beta$, and the barrier-free period is $1-\beta, \beta \in[0,1]$. When there are obstacles, the period of RelayCom mode is $\mu \beta, \mu \in[0,1]$, the period of BackCom mode is $\gamma \beta, \gamma \in[0,1]$, and the period of $\mathrm{EH}$ mode is $(1-\mu-\gamma) \beta, \mu+\gamma \in[0,1]$. When there is no obstacle, the period of $\mathrm{EH}$ is $\alpha(1-\beta), \alpha$ $\epsilon[0,1]$, and the period of BackCom is $(1-\alpha)(1-\beta)$. When there is an obstacle between PT and CR, the signals are interrupted, as shown in the red signal path in Figure 3. At this point, eBD needs to relay and forward the signal of PT to $\mathrm{CR}$, as shown in Figure 3 by the green long dotted line. The backscatter signal path is shown by the green short dotted line, and $\zeta$ is the channel gain.

3.1. EH Mode. Since the eBD is always exposed to PT radiation, it has objective conditions for harvesting RF energy to fulfill the energy demand of relay transmission. When there is no obstacle, eBD transmit its data in BackCom mode or harvest energy from ambient RF signal to prepare for RelayCom mode. When there are obstacles and the energy collected by eBD is insufficient to maintain its internal circuit consumption, backscatter communication is needed to assist relay communication or harvest energy.
According to [17] Friis formula, the power harvested by eBD from the RF signal sent by $\mathrm{PT}$ in free space can be obtained.

$$
P_{\mathrm{H}}=\tau P_{T} \frac{G_{T} G_{R} \lambda^{2}}{(4 \pi d)^{2}}
$$

where $P_{\mathrm{H}}$ is the power harvested by eBD, $P_{T}$ is the transmit power of PT, and $\tau \in[0,1]$ is the efficiency of EH. $G_{T}$ is the antenna gain of PT, $G_{R}$ is the antenna gain of eBD, $\lambda$ is the emission wavelength, and $d$ is the distance between PT and eBD. Throughout the $\mathrm{EH}$ period $(1-\mu$ $-\gamma) \beta+\alpha(1-\beta)$, the energy harvested by $\mathrm{eBD}$ is

$$
E_{h}=[(1-\mu-\gamma) \beta+\alpha(1-\beta)] P_{\mathrm{H}} .
$$

Substituting (1) into (2), $E_{h}$ can be rewritten as

$$
E_{h}=[(1-\mu-\gamma) \beta+\alpha(1-\beta)] \tau P_{T} \frac{G_{T} G_{R} \lambda^{2}}{(4 \pi d)^{2}} .
$$

From (1)-(3), in the whole time unit, the energy harvested by $\mathrm{eBD} E_{h}$ is expressed as a function of three variables $\alpha, \mu$, and $\gamma$. When $\alpha$ increases, $E_{h}$ increases, and when $\mu+\gamma$ increases, $E_{h}$ decreases, but $\alpha$ has an upper limit, which must ensure the time demand of backscatter communication when there is no obstacle. If $\mu+\gamma$ is too small will affect the throughput of relay communication and backscatter communication in obstacle environments, therefore, the value of the three variables $\alpha, \mu$, and $\gamma$ need to be weighed comprehensively. 


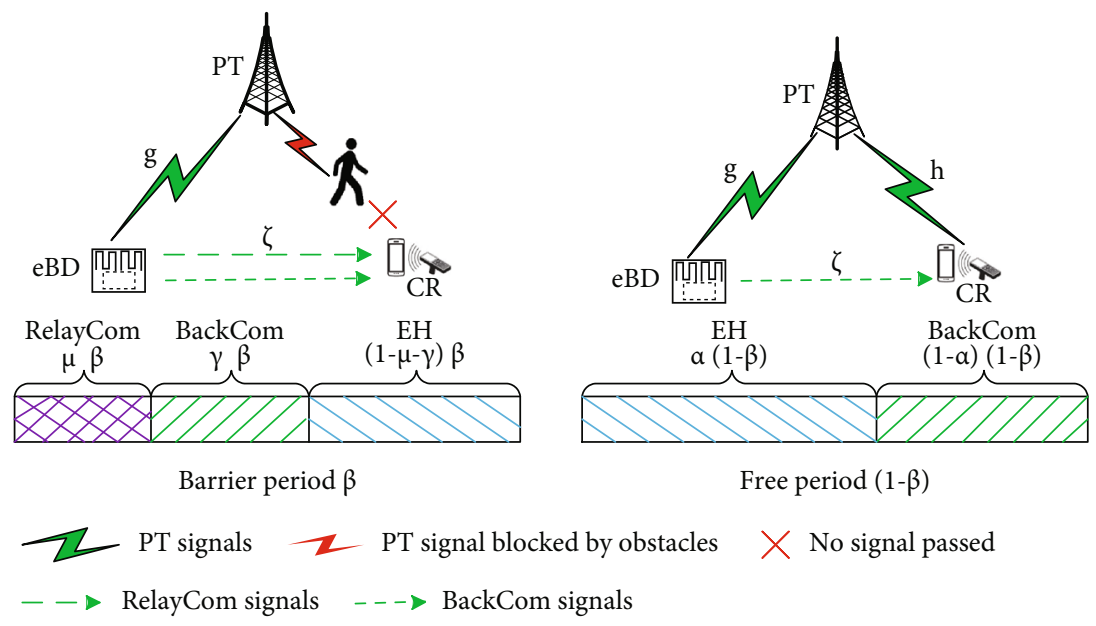

FIGURE 3: Time allocation of ambient backscatter-assisted wireless relay.

3.2. RelayCom Mode. RelayCom mode mainly occurs when there are obstacles. If the stored energy of the eBD exceeds its circuit consumption, it will forward the data directly to CR. Otherwise, eBD will harvest energy or send data through backscatter mode to ensure the effectiveness of signal transmission. eBD transmits data using the harvested energy (excluding the circuit consumption of the eBD itself) in the data transmission period $\mu \beta$. The transmission power of the eBD is

$$
P_{t}=\frac{E_{\mathrm{h}}-E_{\mathrm{c}}}{\mu \beta}, E_{\mathrm{h}} \geq E_{\mathrm{c}} \geq 0,
$$

where $P_{t}$ is the transmission power of eBD in the data transmission period $\mu \beta$, and $E_{\mathrm{c}}$ is the circuit energy loss in the period of $\mu \beta$. Both $E_{\mathrm{h}}$ and $P_{t}$ are nonnegative.

Due to $E_{\mathrm{h}} \geq E_{\mathrm{c}}$, According to formula (2), $[(1-\mu-\gamma) \beta$ $+\alpha(1-\beta)] P_{\mathrm{H}} \geq E_{\mathrm{c}}$, due to the energy consumption of the circuit itself, the period $s$ of $\mathrm{EH}$ during eBD relay communication is expressed as

$$
s=(1-\mu-\gamma) \beta+\alpha(1-\beta) \geq \frac{E_{\mathrm{c}}}{P_{\mathrm{H}}} .
$$

The minimum value of $s$ is defined as $s_{\min }$; therefore,

$$
s_{\min }=\frac{E_{\mathrm{c}}}{P_{\mathrm{H}}} .
$$

Ins $\geq s_{\min }$, in RelayCom, $s_{\min }$ represents the minimum $\mathrm{EH}$ time to ensure the energy consumption of eBD circuit. Since $s \leq 1$, therefore, $s_{\min } \leq 1$.

According to reference [18], the data transmission rate per unit time in the RelayCom mode is expressed as

$$
t_{r}=\eta B \log _{2}\left(1+\frac{P_{t}}{P_{0}}\right)+\tilde{\omega}_{b}(n)
$$

where $\eta \in[0,1]$ is the efficiency of data transmission, $B$ is the bandwidth of the primary channel, $P_{0}=N_{0} / \zeta$ is the ratio of noise power $N_{0}$, and the channel gain coefficient $\zeta, \tilde{\omega}_{b}(n)$, is zero-mean additive Gaussian white noise (AWGN) with variance $N_{\omega b}$. Equation (7) is rewritten as

$$
t_{r}=\eta B \log _{2}\left(1+\frac{P_{t} \zeta}{N_{0}}\right)+\tilde{\omega}_{b}(n) .
$$

Therefore, in RelayCom mode, in the period $\mu \beta$, the number of bits transmitted is

$$
T_{\mathrm{r}}=\mu \beta \eta B \log _{2}\left(1+\frac{P_{t}}{P_{0}}\right)+\tilde{\omega}_{b}(n) .
$$

Substituting (4) into (9), $T_{\mathrm{r}}$ can be rewritten as

$$
T_{\mathrm{r}}=\mu \beta \eta B \log _{2}\left(1+\frac{E_{\mathrm{h}}-E_{\mathrm{c}}}{\mu \beta P_{0}}\right)+\tilde{\omega}_{b}(n) .
$$

Substituting $P_{0}=N_{0} / \zeta$ into (10), $T_{\mathrm{r}}$ can be rewritten as

$$
T_{\mathrm{r}}=\mu \beta \eta B \log _{2}\left(1+\frac{\left(E_{\mathrm{h}}-E_{\mathrm{c}}\right) \zeta}{\mu \beta N_{0}}\right)+\tilde{\omega}_{b}(n) .
$$

From (2)-(10), the expression for $T_{\mathrm{r}}$ is

$$
\begin{aligned}
T_{\mathrm{r}}= & \mu \beta \eta B \log _{2}\left[\mu\left(\beta P_{0}-\beta P_{\mathrm{H}}\right)-\gamma \beta P_{\mathrm{H}}+\alpha(1-\beta) P_{\mathrm{H}}\right. \\
& \left.+\beta P_{\mathrm{H}}-E_{\mathrm{c}}\right]-\mu \beta \eta B \log _{2} \mu \beta P_{0}+\tilde{\omega}_{b}(n) .
\end{aligned}
$$

From (4)-(12), we find that the throughput $T_{\mathrm{r}}$ of RelayCom mode is a function of three variables about $\alpha, \mu$, and $\gamma$, and it increases with the increases of $\alpha$ and decreases with the increases of $\gamma$. Because the RelayCom mode is limited by energy when transmitting data, its throughput increases with the increases of $\alpha$ first, and when the $\mu$ increases to a certain value, $T_{\mathrm{r}}$ starts to decrease. Therefore, to make $T_{\mathrm{r}}$ 
get the maximum value, its variable should take the appropriate value.

3.3. BackCom Mode. When there are obstacles, and the energy harvested by eBD is insufficient to support its internal circuit consumption, the backscatter communicationassisted relay is required to forward the information from PT to CR. When there are no obstacles, PT can directly send information to CR without the relay function of eBD. At this point, $\mathrm{eBD}$ needs to backscatter its data to $\mathrm{CR}$ in a passive way or collect energy.

To obtain the closed expression of ambient backscatter throughput, the premise is that the receiver can sample, demodulate, and decode the received signal. The amount of data transmitted by ambient backscatter in period $(1-\beta$ )$(1-\alpha)+\gamma \beta$ is

$$
T_{b}=(1-\beta)(1-\alpha) R_{b}+\gamma \beta R_{b}+\tilde{\omega}_{b}(n),
$$

where $\gamma \beta$ is the period the eBD transmits data through the backscatter mode when there are obstacles. $(1-\beta)(1-\alpha)$ is the period when eBD transmits itself data through back- scatter communication when there is no obstacle. Backscatter communication has the performance of passive backscatter data. It is a green communication mode. It does not need to generate RF signals actively. Its circuit does not need to use more energy-consuming analog circuit components (such as mixer, amplifier, filter and oscillator), and the energy consumption is almost zero. Therefore, the energy consumption of the circuit is not necessary to be considered in the BackCom mode. At the same time, the interference of $\mathrm{eBD}$ to the main receiver does not need to be considered. The field test has been done in the literature [19], and it is proved that the main receiver will not produce any obvious error owing to the backscatter transmitter unless their distance is less than 7 inches.

The above is a detailed description of the three communication modes of backscatter-assisted wireless relay communication in obstacle environments. Next, the total throughput expression of the system will be given for the three communication modes.

The probability of obstacle and accessibility is $\beta$ and 1 $-\beta$. The segmented expression of a single eBD transmission rate can be expressed as (14)

$$
T= \begin{cases}\mu \beta \eta B \log _{2}\left[\mu\left(\beta P_{0}-\beta P_{\mathrm{H}}\right)-\gamma \beta P_{\mathrm{H}}+\alpha(1-\beta) P_{\mathrm{H}}+\beta P_{\mathrm{H}}-E_{\mathrm{c}}\right]-\mu \beta \eta B \log _{2} \mu \beta P_{0}+\tilde{\omega}_{b}(n), & \text { period of } \mu \beta, \\ (1-\beta)(1-\alpha) R_{b}+\gamma \beta R_{b}+\tilde{\omega}_{b}(n), & \text { period of }(1-\beta)(1-\alpha)+\gamma \beta, \\ 0, & \text { otherwise } .\end{cases}
$$

As shown in formula (14), in a time unit, the throughput of a single eBD can be expressed in three segments. When there are obstacles and $E_{\mathrm{h}}>E_{\mathrm{c}}$, the data transmission mode of eBD is RelayCom mode. The data transmission rate is shown in the first line of the formula (14). When there are no obstacles, or there are obstacles but $E_{\mathrm{h}} \leq E_{\mathrm{c}}$, the data transmission rate of eBD is shown in the second line of the formula (14). Otherwise, the data transmission rate of $\mathrm{eBD}$ is 0 as shown in the third line of the formula (14).

\section{Optimization Model of Three Working Modes}

The goal of this paper's study is to maximize the throughput of the secondary system on the premise of ensuring the quality of service (QoS) of all users. In Section 3, the expression of eBD transmission rate has been obtained. Next, the three working modes are optimized to find their balance point, that is to find out the values of $\alpha, \mu$, and $\gamma$ to maximize the eBD transmission rate.

In one time unit, the transmission rate of a single $\mathrm{eBD}$ is the sum of the transmission rates of RelayCom mode and
BackCom mode, which needs to be considered comprehensively. The total transmission rate is

$$
\begin{aligned}
T(\alpha, \mu, \gamma)= & T_{r}+T_{b}=\mu \beta \eta B \log _{2}\left[\mu\left(\beta P_{0}-\beta P_{\mathrm{H}}\right)\right. \\
& \left.-\gamma \beta P_{\mathrm{H}}+\alpha(1-\beta) P_{\mathrm{H}}+\beta P_{\mathrm{H}}-E_{\mathrm{c}}\right] \\
& -\mu \beta \eta B \log _{2} \mu \beta P_{0}+(1-\beta)(1-\alpha) R_{b} \\
& +\gamma \beta R_{b}+\tilde{\omega}_{b}(n) .
\end{aligned}
$$

To find the values of the three parameters $\alpha, \mu$, and $\gamma$ that maximize $T(\alpha, \mu, \gamma)$, we begin by analyzing the value range and variation relationship of the three parameters. For more intuitive display, the approximate change curve is drawn in Figure 4.

The relational constraint of variables $\mu$ and $\gamma$ along with $\alpha$ changing is shown in Figure 4 , in which the red and yellow curves represent variables $\mu$ and $\gamma$ along with variable $\alpha$ changing, respectively. The variation curves of both variables $\mu$ and $\gamma$ are between 0 and 1 , and the value of $\mu+\gamma$ is also between 0 and 1. First, look at the change of the red curve $\mu$, and when the value of $\alpha$ is close to 0 , the value of $\mu$ is 0 . This is because $\mathrm{eBD}$ has the problem of internal consumption in the circuit when transmitting data through 
RelayCom mode. Along with the increase of $\alpha$, the value of $\mu$ increases rapidly until it is close to 1 . Whether the change curve of $\mu$ will decline depends on the specific value of $\beta$ in the specific environment. Then, look at the changes of the yellow curve $\gamma$, and when the value of $\alpha$ is close to 0 , the value of $\gamma$ is close to 1 . This is because the RelayCom mode is not started during this period, data can only be transmitted in BackCom mode, along with the increase of $\alpha$, and the value of $\gamma$ decreases rapidly until it approaches 0 .
Because the RelayCom mode adopts active communication whereas the BackCom mode adopts passive communication, the RelayCom rate is significantly higher than the BackCom rate. The existence of the maximum communication rate is first considered in this article. Because the additive white Gaussian noise $\tilde{\omega}_{b}(n)$ does not affect the solution of the three parameters $\alpha, \mu$, and $\gamma$, therefore, it can be omitted first. To maximize the transmission rate of $\mathrm{eBD}$ is to find the maximum value of $T(\alpha, \mu, \gamma)$. Firstly, it is proved that there is a maximum value of $T(\alpha, \mu, \gamma)$.

From (15), we have

$$
\frac{\partial T(\alpha, \mu, \gamma)}{\partial \alpha}=\frac{\mu \beta \eta B(1-\beta) P_{\mathrm{H}}}{\left[\mu\left(\beta P_{0}-\beta P_{\mathrm{H}}\right)-\gamma \beta P_{\mathrm{H}}+\alpha(1-\beta) P_{\mathrm{H}}+\beta P_{\mathrm{H}}-E_{\mathrm{c}}\right] \ln 2}-(1-\beta) R_{b} .
$$

Let (16) be equal to 0 , and we have

$$
\mu=\frac{\gamma \beta P_{\mathrm{H}} R_{b} \ln 2-\left[\alpha(1-\beta) P_{\mathrm{H}}+\beta P_{\mathrm{H}}-E_{\mathrm{c}}\right] R_{b} \ln 2}{\left(\beta P_{0}-\beta P_{\mathrm{H}}\right) R_{b} \ln 2-\beta \eta B P_{\mathrm{H}}},
$$

from which, we have that when $\alpha$ is fixed, and $\mu$ is a linear function of $\gamma$; therefore, there is a set of variable values $(\mu$, $\gamma)$ to make the throughput $T(\alpha, \mu, \gamma)$ of eBD to take the maximum value.

From (15), we have

$$
\begin{aligned}
\frac{\partial T(\alpha, \mu, \gamma)}{\partial \mu}= & \beta \eta B \log _{2}\left[\mu\left(\beta P_{0}-\beta P_{\mathrm{H}}\right)-\gamma \beta P_{\mathrm{H}}+\alpha(1-\beta) P_{\mathrm{H}}+\beta P_{\mathrm{H}}-E_{\mathrm{c}}\right] \\
& +\mu \beta \eta B \frac{\left(\beta P_{0}-\beta P_{\mathrm{H}}\right)}{\left[\mu\left(\beta P_{0}-\beta P_{\mathrm{H}}\right)-\gamma \beta P_{\mathrm{H}}+\alpha(1-\beta) P_{\mathrm{H}}+\beta P_{\mathrm{H}}-E_{\mathrm{c}}\right] \ln 2}-\mu \beta \eta B \log _{2} \mu \beta P_{0}-\eta B \frac{\beta P_{0}}{P_{0} \ln 2}
\end{aligned}
$$

Let (18) be equal to 0 , and we have

$$
\begin{aligned}
& \beta \eta B \log _{2}\left[\mu\left(\beta P_{0}-\beta P_{\mathrm{H}}\right)-\gamma \beta P_{\mathrm{H}}+\alpha(1-\beta) P_{\mathrm{H}}+\beta P_{\mathrm{H}}-E_{\mathrm{c}}\right]+\mu \beta \eta B \frac{\left(\beta P_{0}-\beta P_{\mathrm{H}}\right)}{\left[\mu\left(\beta P_{0}-\beta P_{\mathrm{H}}\right)-\gamma \beta P_{\mathrm{H}}+\alpha(1-\beta) P_{\mathrm{H}}+\beta P_{\mathrm{H}}-E_{\mathrm{c}}\right] \ln 2} \\
& \quad-\mu \beta \eta B \log _{2} \mu \beta P_{0}=\eta B \frac{\beta P_{0}}{P_{0} \ln 2},
\end{aligned}
$$

from which, there is a set of variables $(\alpha, \gamma)$ so that the for-

From (15), we have mula is established. The simulation results also verify it.

$$
\frac{\partial T(\alpha, \mu, \gamma)}{\partial \gamma}=\frac{\mu \beta \eta B(-\beta) P_{\mathrm{H}}}{\left[\mu\left(\beta P_{0}-\beta P_{\mathrm{H}}\right)-\gamma \beta P_{\mathrm{H}}+\alpha(1-\beta) P_{\mathrm{H}}+\beta P_{\mathrm{H}}-E_{\mathrm{c}}\right] \ln 2}+\beta R_{b} .
$$




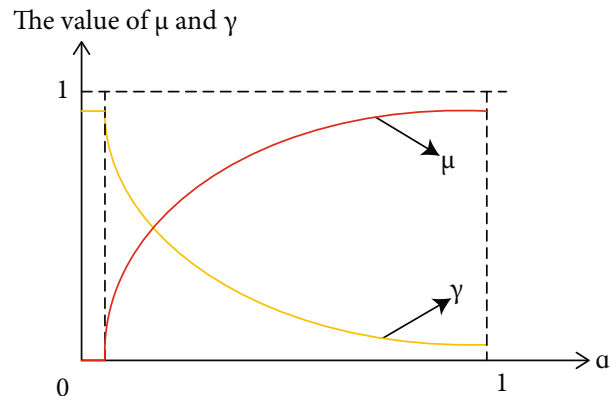

Figure 4: Relational constraint of variables $\mu$ and $\gamma$ along with $\alpha$ changing.

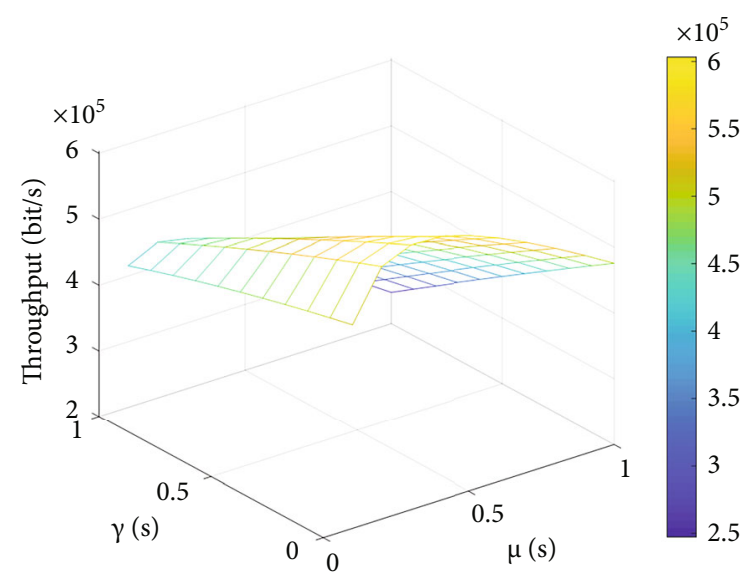

Figure 5: Relationship between throughput and $(\mu, \gamma)$.

Let equation (20) be equal to 0 , and we have

$$
\begin{gathered}
\alpha=\frac{\mu\left[\beta \eta B P_{\mathrm{H}}-R_{b}\left(\beta P_{0}-\beta P_{\mathrm{H}}\right) \ln 2\right]}{(1-\beta) P_{\mathrm{H}} R_{b} \ln 2} \\
+\frac{R_{b}\left(\gamma \beta P_{\mathrm{H}}-\beta P_{\mathrm{H}}+E_{\mathrm{c}}\right) \ln 2}{(1-\beta) P_{\mathrm{H}} R_{b} \ln 2},
\end{gathered}
$$

from which, we have that when $\gamma$ is fixed, and $\alpha$ is a linear function of $\mu$; therefore, there is a set of variable values $(\alpha$, $\mu)$ to make the throughput $T(\alpha, \mu, \gamma)$ of eBD to take the maximum value.

From (15)-(21), we can find that $T(\alpha, \mu, \gamma)$ is a convex function, and there is a set of variable values $(\alpha, \mu, \gamma)$ to make the throughput $T(\alpha, \mu, \gamma)$ of eBD to take the maximum value.

Next, we will look at the special boundary problem. First, when there is no obstacle for a long time $(\beta \approx 0)$, that is, there is no RelayCom, $\mu=0$, then $T(\alpha, \mu, \gamma)=(1-\beta)(1-$ $\alpha) R_{b}$ when $\alpha=0, T(\alpha, \mu, \gamma)$ takes the maximum value, there is no $\mathrm{EH}$ period, the backscattering period is the largest, and eBD transmits data only through backscatter communication mode. The maximum throughput of $\mathrm{eBD}$ is

$$
\max _{\alpha, \mu, \gamma} T(\alpha, \mu, \gamma)=T(\alpha=0)=(1-\beta) R_{b} \approx R_{b}
$$

This occurs when there have been no obstacles for an extended period of time. However, at this time, the energy harvested by $\mathrm{eBD}$ is decreasing, resulting in the reduction of energy reserve, which affects the energy supply of eBD RelayCom mode. Moreover, the relay communication rate of $\mathrm{eBD}$ is greater than the backscatter communication rate; therefore, $\alpha$ is 0 only when there are no obstacles for a long time. When obstacles appear and $E_{\mathrm{h}}<E_{\mathrm{c}}, \alpha$ is closer to 1 ; at this time, the value range of $\mathrm{eBD}$ throughput is

$$
0 \leq T(\alpha, \mu, \gamma) \leq R_{b}
$$

Secondly, obstacles exist for a long time $(\beta \approx 1)$; here, $\alpha$ $\approx 0$. Substituting $\alpha=0$ and $\beta=1$ into (15), the expression of maximum value of $\mathrm{eBD}$ throughput is

$$
\begin{aligned}
\max _{\alpha, \mu, \gamma} T(\alpha, \mu, \gamma) \approx & \mu \eta B \log _{2}\left[\mu\left(P_{0}-P_{\mathrm{H}}\right)-\gamma P_{\mathrm{H}}+P_{\mathrm{H}}-E_{\mathrm{c}}\right] \\
& -\mu \eta B \log _{2} \mu P_{0}+\gamma R_{b},
\end{aligned}
$$

formula (24) is about $\mu, \gamma$, at this time, the value range of eBD throughput is

$$
\begin{aligned}
0 \leq & T(\alpha, \mu, \gamma) \leq \mu \eta B \log _{2}\left[\mu\left(P_{0}-P_{\mathrm{H}}\right)-\gamma P_{\mathrm{H}}+P_{\mathrm{H}}-E_{\mathrm{c}}\right] \\
& -\mu \eta B \log _{2} \mu P_{0}+\gamma R_{b} .
\end{aligned}
$$

Two special boundary problems are given above, and their values are still within the range considered by formula (15), which can be reflected in the simulation. To sum up, the formula (15) of eBD transmission data throughput is applicable regardless of whether there are obstacles or not. The maximum value of $T(\alpha, \mu, \gamma)$ should be $T_{r}+T_{b}$. If the backscatter transmission rate is very high, eBD should communicate data as much as feasible using backscatter. If the backscatter transmission rate is very low, eBD shall harvest energy from the PT signal as much as possible. If the energy harvested is not enough to provide $\mathrm{BDD}$ circuit to transmit data in RelayCom mode when there are obstacles, eBD will choose to transmit data in BackCom mode.

According to the above description, although there are three variables $\alpha, \mu, \gamma$ of the transmission rate $T(\alpha, \mu, \gamma)$ of $\mathrm{eBD}$, there is a certain relationship between variables. Because the basic particle swarm optimization algorithm needs to determine a few parameters, the operation is simple and convenient. When $\beta$ takes the value between 0 and 1 , this paper introduces particle swarm optimization algorithm and solves it according to the following ideas.

Suppose that in a three-dimensional target search space, $m$ particles form a group, because the objective function $T$ in this paper has three variables $\alpha, \mu$, and $\gamma$; therefore, the $i$-th particle is expressed as $X_{i}=\left(x_{i}^{1}, x_{i}^{2}, x_{i}^{3}\right),(i=1,2, \ldots, m)$; that is, the position of the $i$-th particle in three-dimensional space is $X_{i}$, which is a potential solution. We can calculate its fitness value by substituting $X_{i}$ into (15), and the potential solution is measured according to the fitness value. The best 
1. The number of initialization population particles $m=50$, the maximum number of iteration maxItera $=500$, lower limit of position range $\mathrm{lp}=[0,0,0]$, the upper limit up $=[1,1,1]$, the speed limit vmin $=[-1,-1,-1]$, vmax $=[1,1,1]$. The $i$-th particle is expressed as $X_{i}$ $=\left(\mathrm{x}_{i}^{1}, \mathrm{x}_{i}^{2}, \mathrm{x}_{i}^{3}\right)$, substituting it into (15) to calculate its fitness value.

2. Evaluate the fitness of each particle. Particle iteration range

for itera $=1$ : maxItera;

3. For each particle, compare its fitness value $\mathrm{f}_{X_{i}}$ with the fitness value fpbest of the best position pbest it has experienced. If it is larger, take $X_{i}$ as the current best position pbest. For all particles,

if index $=\mathrm{find}\left(\right.$ fpbest $\left.<\mathrm{f}_{X_{i}}\right)$; then

fpbest $=\mathrm{f}_{X_{i}}$;

end if

$$
\text { pbest }=X_{i} \text {; }
$$

The best fitness value and best position of individual history are updated.

4. For each particle $X_{i}$, its fitness value $\mathrm{f}_{X_{i}}$ is compared with the fitness value fgbest of the best position gbest experienced by all

particles. If it is larger, it is regarded as the best position gbest of all particles at present to group.

If fgbest $<\mathrm{f}_{X_{i}}$; then

$$
\begin{aligned}
& \text { fgbest }=\mathrm{f}_{X_{i}} ; \\
& \text { gbest }=X_{i} ;
\end{aligned}
$$

\section{end if}

The best fitness value and best position of population history are updated.

5. Record the historical best position and best fitness value of the population.

recg(itera $)=$ gbest;

recfg(itera) $=$ fgbest;

end for

6. Update the velocity and position of the particles according to (26) and (27).

7. Return to step 2 until the given maximum number of iterations is reached.

Algorithm 1: Algorithm of three parameter selection and maximum throughput solving.

position it has experienced is recorded as pbest $=\left(p_{i}^{1}, p_{i}^{2}, p_{i}^{3}\right)$, and the best position experienced by all particles of the whole population is recorded as gbest $=\left(p_{g}^{1}, p_{g}^{2}, p_{g}^{3}\right)$. The velocity of particle $i$ is expressed as $V_{i}=\left(v_{i}^{1}, v_{i}^{2}, v_{i}^{3}\right)$. Velocity and position of the particle $i$ update formula are [20]

$$
\begin{aligned}
& v_{i}^{d}=w v_{i}^{d}+c_{1} r_{1}\left(p_{i}^{d}-x_{i}^{d}\right)+c_{2} r_{2}\left(p_{g}^{d}-x_{i}^{d}\right), \\
& x_{i}^{d}=x_{i}^{d}+v_{i}^{d},
\end{aligned}
$$

where $i=1,2, \ldots, m$ and $d=1,2,3, w$, are a nonnegative number, called the inertia factor. Learning factor $c_{1}$ and $c_{2}$ is nonnegative constant, and $r_{1}$ and $r_{2}$ are random number varying within the range of $[0,1]$. The first part of $v_{i}^{d}$ is $w$ $v_{i}^{d}$ which is the previous velocity of the particle. The second part of $v_{i}^{d}$ is $c_{1} r_{1}\left(p_{i}^{d}-x_{i}^{d}\right)$, which is the "cognition" part, indicating the thinking of the particle itself. It can be interpreted as that a strengthened random behavior is more likely to appear in the future, and it is assumed that the acquisition of correct knowledge is strengthened. Such a model assumes that the particle is excited to reduce the error. The third part of the $v_{i}^{d}$ is $c_{2} r_{2}\left(p_{g}^{d}-x_{i}^{d}\right)$, which is the "social" part, which indicates the information sharing and cooperation among particles. It can be explained that the particle itself is imitated by other particles; that is, it can be used as a reference for the motion of particles in a population.

The parameters of the algorithm mainly include population size $m$, inertia weight $w$, acceleration constant $c_{1}$ and $c_{2}$, maximum speed $V_{\max }$, and a large number of iteration max-
Itera. Inertia weight makes the particle maintain the inertia of motion, allowing it to expand its search space and explore new areas. Let $w=1$ and acceleration constant $c_{1}=c_{2}=2$, which represents the weight of the statistical acceleration term that pushes each particle to the pbest and gbest positions. When the weight value is low, the particles are allowed to wander outside the target area before being pulled back. While when the weight value is high, the particles will suddenly rush to or cross the target area. $V_{\max }$ determines the accuracy of the solution interval, and the design size is appropriate to avoid particles flying over good positions or falling into local optimization, $v_{i}^{d} \in\left[-v_{\max }^{d}, v_{\max }^{d}\right]$; that is, the velocity of a particle $V_{i}$ is limited by a maximum velocity $V_{\max }=\left(v_{\max }^{1}, v_{\max }^{2}, v_{\max }^{3}\right) \cdot V_{\max }$ is a constant, which is set according to the optimization problem in this paper.

The algorithm for solving the maximum throughput of eBD and its corresponding variable value is as follows.

\section{Numerical Results}

In this section, we illustrate the effectiveness of backscatterassisted wireless relay communication in an obstacle environment through simulation. We assume that the efficiency $\eta$ of data transmission in the RelayCom mode of eBD is 0.7 , the signal bandwidth $B$ is $20 \mathrm{MHz}$, circuit energy loss $E_{\mathrm{c}}$ in cycle $\mu$ is $0.001 \mathrm{~W}$, and additive Gaussian white noise $\tilde{\omega}_{b}(n$ ) is negligible in the performance analysis. Considering that the RelayCom mode needs to consume more energy, the $\mathrm{EH}$ period $\alpha$ of eBD in an obstacle-free environment can be set different values according to the amount of transmission 


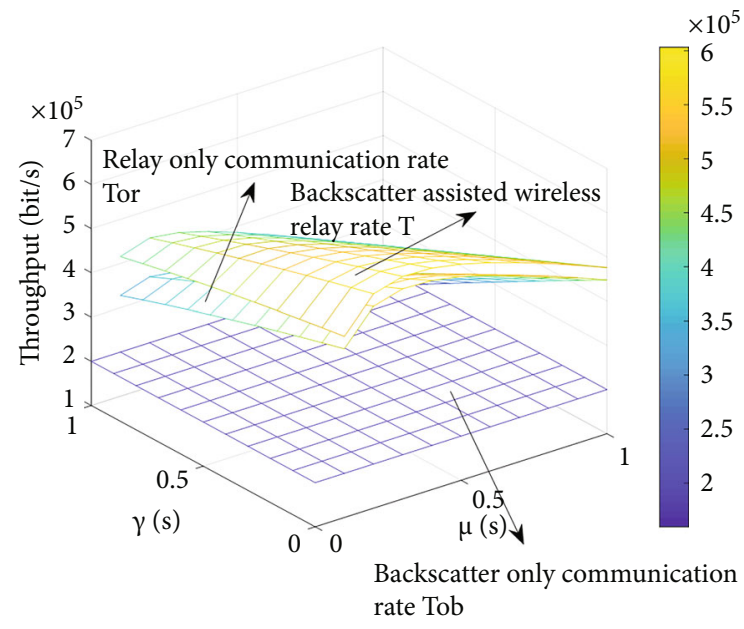

Figure 6: Rate comparison of the backscatter-assisted wireless relay, relay-only communication, and backscatter-only communication.

information required by BackCom. Simultaneously, to visualize the change of an objective function with variables in three-dimensional space, a variable can be fixed without affecting the experimental properties.

In two modes of RelayCom and BackCom, firstly, we study the relationship between the total transmission rate $T$ of eBD and three parameters $\alpha, \mu$, and $\gamma$ in a unit period and the maximum achievable transmission rate $T$ and the values of the corresponding three parameters $\alpha, \mu$, and $\gamma$. Figure 5 plots a three-dimensional grid diagram of the change between the total transmission rate $T$ of eBD in a unit period and the RelayCom period $\mu$ and BackCom period $\gamma$ when there are obstacles. From this Figure, we find that the total throughput $T$ of eBD changes with the change of RelayCom period and BackCom period. Within the value range of $\mu$ and $\gamma$, the change process of $T$ is a smooth surface, and there is a maximum point of throughput $T$. Therefore, as $\mu$ and $\gamma$ change, the total throughput $T$ will change. We can choose the appropriate RelayCom time and BackCom time according to the changes of actual requirements and conditions, to achieve the maximum system throughput and channel spectral efficiency. Through hypothesis and Algorithm 1, we can get that the values of three parameters: eBD EH period $\alpha$ without obstacles, RelayCom period $\mu$ with obstacles, and BackCom period $\gamma$ with obstacles are $0.8,0.3$, and 0 , respectively. The corresponding eBD total throughput $T$ is $6.0321 \times 10^{5} \mathrm{bit} / \mathrm{s}$. In Figure 5, the throughput values are represented by yellow, green, and blue color gradients from large to small.

Next, we study how system throughput varies in three cases: backscatter-assisted wireless relay in obstacle environment, relay-only communication, and backscatter-only communication. To facilitate observation, we put the figures of system throughput changing with parameters of the three cases in the same simulation figure. At the same time, the particle swarm optimization method is employed to calculate the maximum throughput and corresponding parameter values of the three cases. Figure 6 plots the relationship

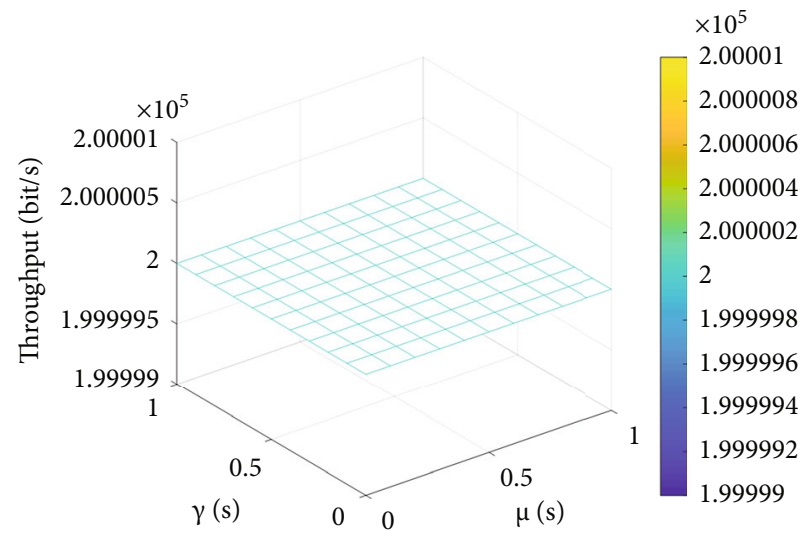

FIgURE 7: Throughput of the backscatter-assisted wireless relay when $\beta=0$.

between throughput $T$ and RelayCom period $\mu$ and BackCom period $\gamma$ in three cases. Figure 6 clearly shows that the throughput of backscatter-assisted wireless RelayCom is the largest. BackCom has the lowest throughput. Between them lies the throughput of wireless RelayCom. Secondly, the throughput of the three cases is a smooth surface, and each has its maximum value. According to Algorithm 1, the maximum throughput $T$ of backscatter-assisted wireless relay is $6.0321 \times 10^{5} \mathrm{bit} / \mathrm{s}$, corresponding to the $\mathrm{EH}$ period $\alpha$ of eBD without obstacles, the RelayCom period $\mu$ with obstacles, and the BackCom period $\gamma$ with obstacles that are $0.8,0.3$, and 0 , respectively. The maximum throughput $T_{\text {or }}$ of wireless relay-only communication is $5.7521 \times 10^{5}$ $\mathrm{bit} / \mathrm{s}$, and the values corresponding to the parameters $\alpha, \mu$, and $\gamma$ are $0.8,0.3$, and 0 , respectively. The maximum throughput $T_{o b}$ of only backscatter communication is $2 \times$ $10^{5} \mathrm{bit} / \mathrm{s}$, and the values corresponding to the three parameters $\alpha, \mu$, and $\gamma$ are 0,0 , and 1, respectively. Figure 6 depicts throughput values as yellow, green, and blue color gradients ranging from large to small.

Next, the special case is analyzed when there is no obstacle between PT and CR $(\beta=0)$. eBD does not need RelayCom and transmits data only through BackCom mode. Figure 7 plots the relationship between system throughput $T$ and RelayCom period $\mu$ and BackCom period $\gamma$ when there are no obstacles for a long time. From Figure 7, we find that the total throughput $T$ of eBD is a constant that does not change with variables, with a size of $2 \times 10^{5} \mathrm{bit} / \mathrm{s}$. This value is less than $6.0321 \times 10^{5} \mathrm{bit} / \mathrm{s}$, and this shows that $\beta=0$ does not affect the maximum throughput of backscatter-assisted wireless relay communication in an obstacle environment. Figure 7 depicts throughput values as yellow, green, and blue color gradients ranging from large to small.

When there is always an obstacle between PT and $\mathrm{CR}(\beta=1)$, eBD can both backscatter data and relay data. As long as the energy harvested by eBD is greater than its circuit consumption, the RelayCom mode can be enabled for communication and its communication rate is substantially faster than BackCom mode. Figure 8 shows the grid diagram of the changing relationship between the throughput $T$ and the RelayCom period $\mu$ and the BackCom period 


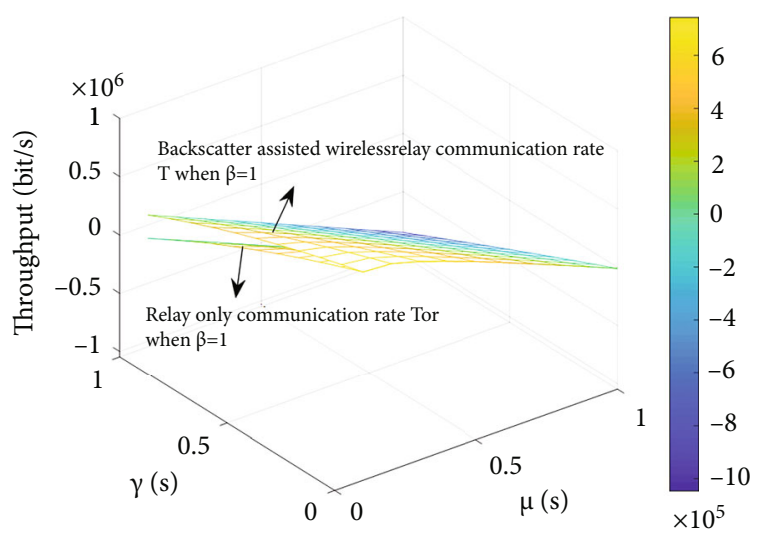

FIGURE 8: Rate comparison of the backscatter-assisted wireless relay and relay-only communication when $\beta=1$.

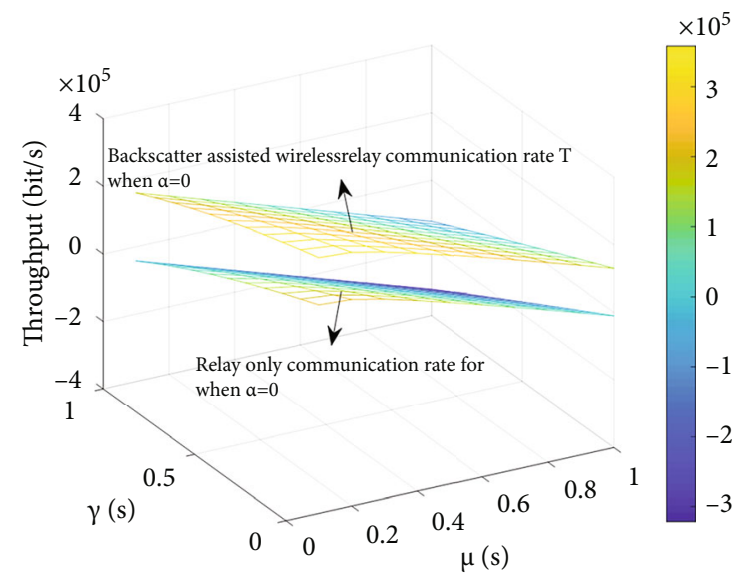

FIGURE 9: Rate comparison of backscatter-assisted wireless relay and relay-only communication when $\alpha=0$.

$\gamma$ when $\beta=1$. From Figure 8 , we find that the total throughput $T$ of eBD changes with the change of $\mu$ and $\gamma$. Within the value range of $\mu$ and $\gamma$, the change process of $T$ shows a smooth surface, and there is a maximum point of throughput $T$. Therefore, with the change of $\mu$ and $\gamma$, the total throughput $T$ will change. To maximise system throughput and channel spectral efficiency, we can adjust the RelayCom and BackCom times in response to changes in actual demand and conditions. Through hypothesis and algorithm 1 , we can conclude that the maximum communication rate of the backscatter-assisted wireless relay is $7.435 \times 10^{5}$ bit/ $\mathrm{s}$, and the corresponding values of the three parameters $\alpha$, $\mu$, and $\gamma$ are $0.8,0.1$, and 0 , respectively. When there is wireless relay-only communication, the maximum communication rate is $7.435 \times 10^{5} \mathrm{bit} / \mathrm{s}$, and the corresponding values of the three parameters $\alpha, \mu$, and $\gamma$ are $0.8,0.1$, and 0 , respectively. As can be seen from Figure 8 , the rate of the backscatter-assisted wireless relay is greater than that of relay-only communication; however, there is an intersection when $\gamma=0.1$. Figure 8 depicts throughput values as yellow, green, and blue color gradients ranging from large to small.

Figures 7 and 8 , respectively, study $\beta=0$ and $\beta=1$ two boundary cases. Next, we need to study the changes of

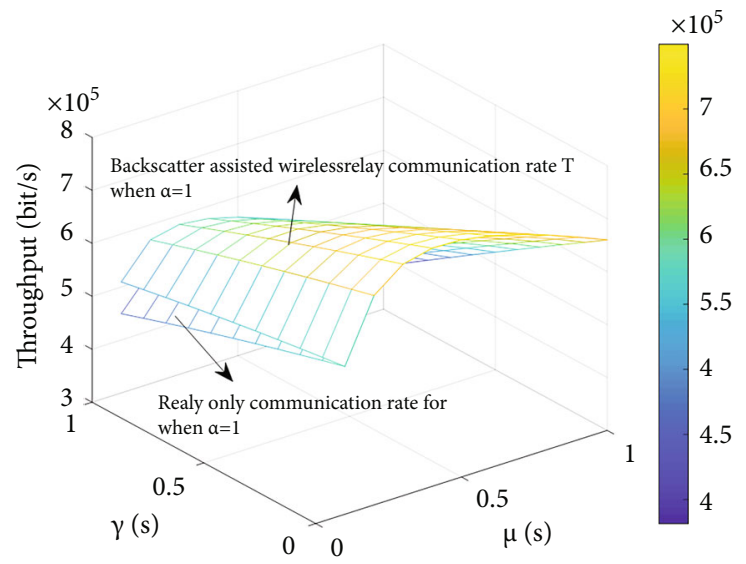

FIgURE 10: Rate comparison of backscatter-assisted wireless relay and relay-only communication when $\alpha=1$.

throughput $T$ and $T_{\text {or }}$ when $\alpha=0$ and $\alpha=1$ under the same variable conditions. Figure 9 shows the relationship between throughput of $T$ and $T_{\text {or }}$ and RelayCom period $\mu$ and BackCom period $\gamma$ when $\alpha=0$. As can be seen from Figure 9, when $\alpha=0$, the backscatter-assisted wireless relay communication rate is greater than that when there is relay-only communication. Through hypothesis and Algorithm 1, we can conclude that the maximum backscatter-assisted wireless relay rate is $3.5814 \times 10^{5} \mathrm{bit} / \mathrm{s}$, and the corresponding values of the three parameters $\alpha, \mu$, and $\gamma$ are $0,0.1$, and 0 , respectively. When there is wireless relay-only communication, the maximum rate is $2.1814 \times 10^{5} \mathrm{bit} / \mathrm{s}$, and the corresponding values of the three parameters $\alpha, \mu$, and $\gamma$ are $0,0.1$, and 0 , respectively. Figure 9 depicts throughput values as yellow, green, and blue color gradients ranging from large to small.

Figure 10 shows the relationship between throughput of $T$ and $T_{\text {or }}$ and RelayCom period $\mu$ and BackCom communication period $\gamma$ when $\alpha=1$. As can be seen from Figure 10, when $\gamma=0$, the values of $T$ and $T_{\text {or }}$ are equal. At this time, through hypothesis and Algorithm 1, we can conclude that the values of $T$ and $T_{\text {or }}$ are $7.4971 \times 10^{5} \mathrm{bit} / \mathrm{s}$, and the corresponding values of the three parameters $\alpha, \mu$, and $\gamma$ are $1,0.4$, and 0 , respectively. When $\gamma \neq 0$, the rate of backscatterassisted wireless relay communication is greater than that when there is relay-only communication. Figure 10 depicts throughput values as yellow, green, and blue color gradients ranging from large to small.

Figure 11 shows the performance comparison of backscatter-assisted wireless relay communication, relayonly communication, and backscatter-only communication when $\beta$ takes different values. Among them, the red curve is the change curve of the throughput of backscatterassisted wireless relay communication with $\beta$. The green curve is the change curve of the throughput of relay-only communication with $\beta$. The blue curve is the change curve of the throughput of backscatter-only communication with $\beta$. As can be seen from Figure 11, the throughput of backscatter-assisted wireless relay communication is the largest, followed by relay-only communication, and the throughput of backscatter-only communication is the 


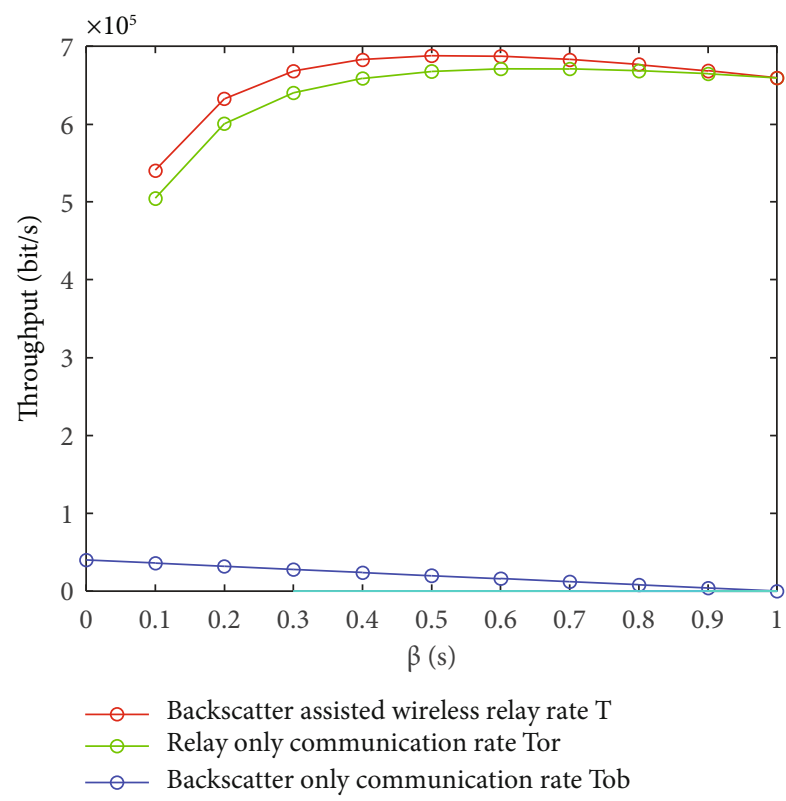

FIGURE 11: Performance comparison of throughput in three cases when $\beta$ takes different values.

smallest. When $\beta=0.5$, the maximum throughput of backscatter-assisted wireless relay communication is 6.8796 $\times 10^{5} \mathrm{bit} / \mathrm{s}$. When $\beta=0.6$, the maximum throughput of relay-only communication is $6.7125 \times 10^{5} \mathrm{bit} / \mathrm{s}$. When $\beta=$ 0 , the maximum throughput of backscatter-only communication is $4.0 \times 10^{4} \mathrm{bit} / \mathrm{s}$.

Figures 9 and 10 show the changes of throughput $T$ and $T_{o b}$ when $\alpha=0$ and $\alpha=1$, respectively. In these two boundary cases, we proved that the transmission rate of backscatter-assisted wireless relay communication is greater than or equal to the transmission rate of relay-only communication. The experimental results corroborate the theoretical research results. We evaluate the effectiveness and superiority of the proposed backscatter-assisted wireless relay communication in an obstacle environment in Figures 5-11. We analyze the optimal time schemes when the three communication modes reach equilibrium, and analytically prove the existence, uniqueness, and stability of this balance. At the same time, the effectiveness of the proposed algorithm is proved. In the obstacle environment, backscatter-assisted wireless relay communication can greatly improve the system throughput and channel spectral efficiency, therefore alleviating spectrum congestion problem to a certain extent. This provides an effective reference for the complexity of the $6 \mathrm{G}$ environment and the shortage of spectrum.

\section{Conclusions}

In this paper, we have studied the backscatter-assisted wireless relay communication network in an obstacle environment. Specifically, to obtain the maximum throughput of eBD communication, we describe the communication mode selection problem of eBD as a particle swarm optimization problem. Through this optimization method, we find the value of three parameters of $\mathrm{EH}$ period $\alpha$ of $\mathrm{eBD}$ when there is no obstacle, period $\mu$ of RelayCom with obstacles, and BackCom period $\gamma$ with obstacles. And we calculate the maximum value of $\mathrm{eBD}$ throughput corresponding to these parameters' value. We have proved that the designed $\mathrm{EH}$, RelayCom, and BackCom communication modes can achieve a unique and stable balance. We investigated the optimal time scheme and proved the effectiveness of the proposed algorithm. Furthermore, we also compare backscatterassisted wireless RelayCom, relay-only communication, and backscatter-only communication. The results show that backscatter-assisted wireless RelayCom has greater throughput and higher spectral efficiency in an obstacle environment. We will lay the backscatter equipment in the simultaneous wireless information and power transfer system to strengthen the transmission of information and energy and further study the deployment of intelligent nodes in the face of dynamic CR in the obstacle environment.

\section{Data Availability}

As the research data in this paper is related to my research topic, the experimental data will not be disclosed temporarily. Thank you.

\section{Conflicts of Interest}

The authors declare that there are no conflicts of interest regarding the publication of this paper.

\section{Authors' Contributions}

This article was jointly completed by the authors Jinfeng Li, Qinyin Ni, and Xiaorong Zhu, from the selection of research sites to the completion of the article. All the authors are very serious. The details are as follows: Jinfeng Li contributed to the conceptualization, methodology, software, data curation, and writing original draft. Qinyin Ni contributed to the visualization, investigation, validation, and editing. Xiaorong Zhu contributed to the supervision, formal analysis, writing reviewing and editing, and funding acquisition.

\section{Acknowledgments}

This work was supported by the Natural Science Foundation of China (92067101), Program to Cultivate Middle-Aged and Young Science Leaders of Universities of Jiangsu Province and Key R\&D Plan of Jiangsu Province (BE20210133), and Jiangsu Province Scientific Research and Practice Innovation Plan Project (No. KYCX20_0720).

\section{References}

[1] R. Duan, E. Menta, H. Yigitler, R. Jantti, and Z. Han, "Hybrid beamformer design for high dynamic range ambient backscatter receivers," in 2019 IEEE International Conference on Communications Workshops (ICC Workshops), pp. 1-6, Shanghai, China, 2019.

[2] Y. Peng, L. Shangguan, Y. Hu et al., "PLoRa: a passive longrange data network from ambient LoRa transmissions,", in 
In Proceedings of the 2018 Conference of the ACM Special Interest Group on Data Communication, pp. 147-160, Budapest, Hungary, 2018.

[3] X. Lu, D. Niyato, H. Jiang, E. Hossain, and P. Wang, "Ambient backscatter-assisted wireless-powered relaying," IEEE Transactions on Green Communications and Networking, vol. 3, no. 4, pp. 1087-1105, 2019.

[4] G. Wang, F. Gao, R. Fan, and C. Tellambura, "Ambient backscatter communication systems: detection and performance analysis," IEEE Transactions on Communications, vol. 64, no. 11, pp. 4836-4846, 2016.

[5] F. Jameel,, T. Ristaniemi, I. Khan, and B. M. Lee, "Simultaneous harvest-and-transmit ambient backscatter communications under Rayleigh fading," EURASIP Journal on Wireless Communications and Networking, vol. 2019, no. 1, Article ID 166, pp. 1-9, 2019.

[6] D. T. Hoang, D. Niyato, P. Wang, D. I. Kim, and Z. Han, "Ambient backscatter: a new approach to improve network performance for RF-powered cognitive radio networks," IEEE Transactions on Communications, vol. 65, no. 9, pp. 36593674, 2017.

[7] S. A. Mousavifar, Y. Liu, C. Leung, M. Elkashlan, and T. Q. Duong, "Wireless energy harvesting and spectrum sharing in cognitive radio," in 2014 IEEE 80th Vehicular Technology Conference (VTC2014-Fall), pp. 1-5, Vancouver, BC, Canada, Sep. 2014.

[8] B. Lyu, H. Guo, Z. Yang, and G. Gui, “Throughput maximization for hybrid backscatter assisted cognitive wireless powered radio networks," IEEE Internet of Things Journal, vol. 5, no. 3, pp. 2015-2024, 2018.

[9] L. Xu, K. Zhu, R. Wang, and S. Gong, "Performance analysis of RF-powered cognitive radio networks with integrated ambient backscatter communications," Wireless Communications and Mobile Computing, vol. 2018, Article ID 8509693, 16 pages, 2018.

[10] X. Gao, S. Feng, D. Niyato, P. Wang, K. Yang, and Y. Liang, "Dynamic access point and service selection in backscatterassisted RF-powered cognitive networks," IEEE Internet of Things Journal, vol. 6, no. 5, pp. 8270-8283, 2019.

[11] J. Li, R. Bhattacharyya, S. Paul, S. Shakkottai, and V. Subramanian, "Incentivizing sharing in realtime D2D streaming networks: a mean field game perspective," IEEE/ ACM Transactions on Networking, vol. 25, no. 1, pp. 3-17, 2017.

[12] X. Lu, D. Niyato, H. Jiang, D. I. Kim, Y. Xiao, and Z. Han, "Ambient backscatter assisted wireless powered communications," IEEE Wireless Communications, vol. 25, no. 2, pp. 170-177, 2018.

[13] N. van Huynh, D. T. Hoang, X. Lu, D. Niyato, P. Wang, and D. I. Kim, "Ambient backscatter communications: a contemporary survey," IEEE Communications Surveys \& Tutorials, vol. 20, no. 4, pp. 2889-2922, 2018.

[14] P. Hu, P. Zhang, M. Rostami, and D. Ganesan, "Braidio: an integrated active-passive radio for mobile devices with asymmetric energy budgets," in Proceedings of the 2016 ACM SIGCOMM Conference, pp. 384-397, Florianopolis, Brazil, Aug. 2016.

[15] X. Lu, P. Wang, D. Niyato, D. I. Kim, and Z. Han, "Wireless networks with RF energy harvesting: a contemporary survey," IEEE Communications Surveys \& Tutorials, vol. 17, no. 2, pp. 757-789, 2015.
[16] H. Chen, Y. Li, J. Luiz Rebelatto, B. F. Uchoa-Filho, and B. Vucetic, "Harvest-then-cooperate: wireless-powered cooperative communications," IEEE Transactions on Signal Processing, vol. 63, no. 7, pp. 1700-1711, 2015.

[17] C. A. Balanis, Antenna Theory: Analysis and Design, John Wiley \& Sons, New York, NY, USA, 2012.

[18] H. Huang and V. K. N. Lau, "Decentralized delay optimal control for interference networks with limited renewable energy storage," IEEE Transactions on Signal Processing, vol. 60, no. 5, pp. 2552-2561, 2012.

[19] V. Liu, A. Parks, V. Talla, S. Gollakota, D. Wetherall, and J. R. Smith, "Ambient backscatter: wireless communication out of thin air," ACM SIGCOMM Computer Communication Review, vol. 43, no. 4, pp. 39-50, 2013.

[20] Y. Wang, Research on Intelligent Nodes Deployment Planning Algorithms for Internet of Things Applications, [M.S. thesis], Nanjing University of Posts and telecommunications, 2020. 